\title{
Flexibility of attention between stimulus dimensions
}

\author{
GLYN W. HUMPHREYS \\ Birkbeck College, University of London, London WCIE 7HX, England
}

\begin{abstract}
Three experiments examined the flexibility of attention to the color and location dimensions of visual stimuli. Displays typically consisted of two curved lines, one a target and the other a distractor. Subjects were precued to the location of the target, and they made a speeded response to that item's direction of curvature. In Experiment 1, a color difference between the stimuli facilitated selective attention to targets. Experiment 2 demonstrated that this effect was not automatic. Although component color differences were necessary for selective attention, they were not sufficient unless they reliably specified the target. Furthermore, when color was informative but the target was in an unexpected color, performance was markedly impaired. A third experiment, using a response-deadline technique, showed that stimulus color was resolved before stimulus location and that attention can be exclusive to the color dimension. The results were interpreted in terms of a model assuming that stimulus color and location are processed independently. Subjects attend to the dimension resolved most quickly which can reliably specify the target.
\end{abstract}

Current views of information processing suggest that performance limitations are due both to the quality of the input and to the quality of the processes that act upon, and whose action is determined by, the input (Allport, 1980; Garner, 1970, 1974; Norman \& Bobrow, 1975). Performance on a given task will depend on the interaction between these factors.

To illustrate this general point, consider the study reported by Pomerantz and Garner (1973). They presented subjects with a sequence of cards, each containing a pair of bracket stimuli positioned in close spatial proximity. A set of such stimuli is shown in Figure 1a. The subjects had to sort the cards on the basis of whether a designated target bracket (e.g., the right-hand bracket in any configuration) was curved to the left, as in the stimuli " $)($ " and " $((, "$ or the right, as in the stimuli "))" and " 0 ." Pomerantz and Garner found that sorting times were slower when the irrelevant (left-hand) bracket was curved randomly across trials than when it was constant. Since these two conditions differ only in the information conveyed by the irrelevant bracket, the result demonstrates that subjects failed to selectively process target elements in these configurations. Following Garner's (1976) terminology, this will be referred to as a configural effect.

The configural effect obtained by Pomerantz and Garner (1973) suggests a lack of spatial selectivity in

An earlier version of this paper was presented at the meeting of the Experimental Psychology Society at Cambridge, England, in July 1980. The author wishes to thank Lindsay Evett for her comments on the paper and an anonymous reviewer who drew my attention to the possibility of criterion differences between the groups in Experiment 1. our processing of visual stimuli. Eriksen and his colleagues (e.g., B. A. Eriksen \& C. W. Eriksen, 1974; C. W. Eriksen, Hamlin, \& Daye, 1973; C. W. Eriksen \& Hoffman, 1972, 1973) have proposed that spatial selectivity is limited within an area of about $1 \mathrm{deg}$ of visual angle around attended locations. In line with this proposal, increasing the visual angle between the bracket stimuli in Figure 1 to more than 1 deg enables targets to be selectively processed. Reaction times (RTs) to the curvature of targets do not then differ in the random and the constant distractor conditions (Humphreys, 1981; Pomerantz \& Schwaitzberg, 1975). In this case, improving the quality of the input overcomes limitations in our processing capabilities (due to poor spatial selectivity).

Interestingly, subjects can demonstrate some control over the selectivity of visual attention in the appropriate circumstances. When they have foreknowledge of and can fixate a target location, there is selective processing even within the seemingly critical area. For instance, Humphreys (1981) has shown that the configural effect of Pomerantz and Garner (1973) does not occur when subjects fixate the target location. This was so, even when the distractor was only $.5 \mathrm{deg}$ away from the target. This suggests that subjects adopt a narrower span of visual attention when a target is expected at fixation than when it is expected away from fixation.

These results indicate that selective visual processing can be determined both by the properties of the stimulus (e.g., the spatial separation of targets and distractors) and by the process set (cf. Duncan, 1980) adopted by the observer (e.g., the span of visual attention).

The above studies investigated the interaction be- 
a

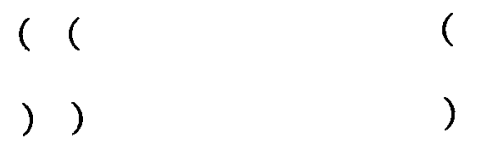

$\mathrm{b}$

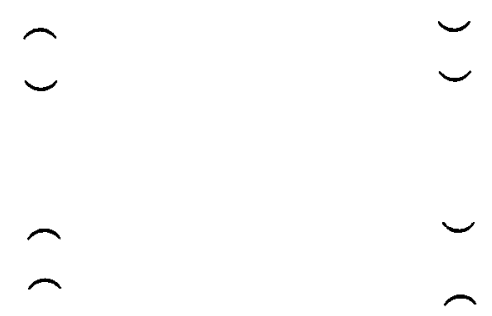

Figure 1. (a) The stimulus sets used by Pomerantz and Garner (1973) and by Pomerantz and Schwaitzberg (1975). (b) The samecolor stimulus sets used in Conditions $R$ and $C$ in Experiment 1.

tween stimulus-determined and perceiver-determined factors when stimuli were defined solely on the basis of their relative locations. The present experiments sought to extend this work by examining the interactions between these factors when stimuli differed along a second physical dimension, that of color. The study is based on the configural effect of Pomerantz and Garner (1973), but varies target and distractor stimuli along the color as well as the location dimension.

Interest focuses on two current models dealing with the processing of multidimensional visual stimuli, both of which provide elegant explanations of the finding that selective attention is facilitated when targets are specified by their color as well as their location (e.g., Allport, 1971; Chen \& Allnutt, 1977; Clark, 1969; Williams, 1966). The holistic processing model of Lockhead and his associates (Lockhead, 1966, 1970, 1972; Lockhead \& King, 1977) proposes that stimuli are initially represented as unitary configurations in multidimensional, euclidian, psychological space. The facility of selective attention within any configuration is assumed to be a function of the discriminability of the components in this space. Additionally, it is assumed that loci in the space are correlated with the structural similarity of stimulus components. Thus, components that are the same color will be represented as closer to one another than will components differing in color. Selective attention to targets will be more efficient in the latter than in the former situation. Alternatively, the independent dimensional processing model proposes that at least some stimulus dimensions (e.g., color and location) can be analyzed independently and in parallel (Biederman \& Checkosky, 1970; Dykes, 1979; Dykes \& Cooper, 1978; Garner, 1974, 1976; Hawkins, 1969). In this case, a stimulus component could be attended on the basis of the first dimension to be processed which defines the target (cf. Biederman \& Checkosky, 1970; C. W. Eriksen \& Schultz, 1979). If there is an overlap in the resolution times for the relevant dimensions, targets specified by both their color and their location should be attended more efficiently than targets specified only by their location.

Experiment 1 here was undertaken to confirm that a reliable color difference between targets and distractor stimuli enables subjects to selectively attend to targets. Experiments 2 and 3 tested between the above accounts of the selective processing effect. They also examined the degree of control a perceiver can effect over stimulus processing, since any model of perception must clarify the role of stimulusdetermined and perceiver-determined factors (see Nickerson, 1978).

\section{EXPERIMENT 1}

\section{Method}

Subjects. There were 16 paid volunteer subjects from the Birkbeck College subject pool ( 7 male, 9 female). All participants had normal or corrected-to-normal vision, and each subject took part in three sessions: two experimental sessions, each lasting about $30 \mathrm{~min}$, and a control session lasting about $50 \mathrm{~min}$.

Apparatus. Stimuli were presented in a three-field Electronics Development tachistoscope. The luminance of the fixation and display fields was $5 \mathrm{fL}$, measured by a sport photometer. Subjects initiated a trial by depressing a handswitch with the nonpreferred hand, and this also started a Birkbeck Laboratory timer. Responses were made to the direction of curvature of the target line by moving a lever with the preferred hand. The direction of movement was compatible with the required response ("up" vs. "down"). The lever closed a relay to the timer, enabling RT to be recorded. The experimenter was informed of the response category by the illumination of a light on the timer.

Stimuli. The stimuli were the same as those used by Pomerantz and Garner (1973) and Pomerantz and Schwaitzberg (1975), except that they were projected in the vertical rather than the horizontal meridian (see Figure 1b for examples of the target-distractor pairs used in Conditions $\mathbf{R}$ and $\mathbf{C}$ here). Stimuli were drawn with the Staedtler Mars $57207 \mathrm{M}$ stencil and the Staedtler 07M pen. Target lines were precued by a black horizontal line of the same length and thickness as the stimulus lines, which appeared at the target location in the visual field. Each line subtended a horizontal visual angle of $.93 \mathrm{deg}$ and a vertical visual angle of $.12 \mathrm{deg}$ from the viewing distance of $508 \mathrm{~mm}$. The fixation field contained a central black cross.

Procedure. Subjects were instructed to initiate the presentation cycle when fixated. When the cycle was onset, the location marker was presented for $50 \mathrm{msec}$, during which the fixation field was inhibited. This returned at the location marker offset for $200 \mathrm{msec}$. The stimulus field was then presented for $\mathbf{2 0 0} \mathrm{msec}$, during which the fixation field was again inhibited. At the stimulus field offset, the fixation field returned and remained on during the intertrial interval. Previous work had shown that the stimulus-onset asyn- 
chrony of $250 \mathrm{msec}$ between the location marker and the stimulus field was sufficient for subjects to process and attend to the precue (Colegate, Hoffman, \& C. W. Eriksen, 1973; Humphreys, 1981).

The subjects were told to attend to the location of the precuing line while remaining centrally fixated, and they responded to the curvature of the line that appeared in the cued location. The target line could appear at a visual angle of $.25 \mathrm{deg}$ randomly above or below fixation. The distractor line was presented $.25 \mathrm{deg}$ on the opposite side of fixation to the target on a given trial. The subjects were notified by the experimenter if they made any errors.

Design. The color-congruence of the bracket stimuli was varied between subjects. For the same-color subject group, target and distractor lines were always identical in color. For half these subjects they were always red, and for the other half they were always black. For the different-color subject group, the bracket stimuli always differed in color. For half these subjects the target was always red and the distractor black, and for the other half the target was always black and the distractor red.

Every subject took part in four conditions: (1) random (R)-the distractor was curved either up or down at random; (2) constant (C) - the distractor was always curved either up or down across a block of trials; (3) straight-line control (S)-the distractor was a straight line; and (4) no distractor control $(\mathrm{N})$-no distractor was presented.

The critical test of selective attention to target stimuli is provided by the contrast between Conditions $R$ and $C$ (Pomerantz \& Garner, 1973). Performance should be worse in Condition $R$ than in Condition $C$ if subjects are unable to selectively attend to the target. Furthermore, if there is a tendency for reliable color differences between targets and distractors to facilitate selective attention, the difference between these test conditions should be smaller for the different-color group than for the same-color group.

The two control conditions ( $\mathrm{S}$ and $\mathrm{N}$ ) served to test for any facilitation or impairment of target processing produced by the distractor. The baseline for responding to the target without any context is provided by the no-distractor control, N. However, any differences between the experimental treatments ( $R$ and $C$ ) and this condition could be due to lateral masking between the stimulus components (Matthews, 1974; Shaw, 1969). In order to control for this possibility, the straight-line distractor condition, $\mathrm{S}$, was included.

The two experimental conditions were administered independently, and the control treatments were presented in a third session. The order of presentation of the sessions was counterbalanced over each subject group, and the order of presentation of the control conditions was counterbalanced over the order of sittings. There were 128 trials in each condition $(R, C, S$, and $N$ ), in which the target was presented above and below fixation and was curved up and down an equal number of times ( 32 trials for each combination). The order of presentation was pseudorandomized for each session undertaken by each subject.

\section{Results}

The mean RTs for each subject in each condition were collapsed. Responses with RTs \pm 3 standard deviations from each subject's mean latency in each condition were discarded along with all errors. Table 1 contains the mean correct RTs and percentage errors on red and black targets in each color congruity group in each condition.

A preliminary ANOVA showed that there was no difference in $\mathrm{RT}$ to targets above and below fixation $(F<1.0)$ and that this variable did not interact with either the condition or the subject group factor (both $F<1.0$ ). A null effect of field of target presentation was also found in Experiments 2 and 3, and the variable will therefore be omitted from further consideration.

RTs were analyzed with performance in the experimental conditions ( $R$ and $C$ ) separated into trials when the target and distractor, respectively, matched and mismatched in orientation. The data were submitted to an ANOVA for a mixed design with one within-subjects factor (Conditions $\mathbf{R}$ matching, $\mathbf{R}$ mismatching, $C$ matching, $C$ mismatching, $S$, and $N$ ) and two between-subjects factors (congruity of component colors and color of target). There were statistically significant main effects of component color congruity $[F(1,12)=53.48, p<.001]$, target color $[F(1,12)=7.53, p<.025]$, and conditions $[F(5,60)=$ $12.51, \mathrm{p}<.001]$. The interaction between the component color congruity and the condition effects was statistically significant $[F(5,60)=5.91, p<.001]$, but the Target Color by Condition interaction failed to reach significance $[F(5,60)=1.07, p>.05]$. None of the other interactions approached significance (all Fs $<1.0$ ).

The Color Congruity by Condition interaction was analyzed in more detail using the Newman-Keuls method. For the same-color subjects, there was a large impairment in Condition $\mathbf{R}$ when the components mismatched in orientation relative to all the

Table 1

Mean Correct Reaction Times (RT) in Milliseconds and Percentage Errors (PE) in Each Color Congruity Group as a Function of the Conditions and the Target Color (TC) in Experiment 1

\begin{tabular}{|c|c|c|c|c|c|c|c|c|}
\hline \multirow[b]{4}{*}{ Condition } & \multicolumn{8}{|c|}{ Color Congruity Group } \\
\hline & \multicolumn{4}{|c|}{ Same Color } & \multicolumn{4}{|c|}{ Different Color } \\
\hline & \multicolumn{2}{|c|}{ TC: Black } & \multicolumn{2}{|c|}{ TC: Red } & \multicolumn{2}{|c|}{ TC: Black } & \multicolumn{2}{|c|}{ TC: Red } \\
\hline & RT & PE & RT & PE & $\mathrm{RT}$ & PE & RT & PE \\
\hline $\begin{array}{l}\mathrm{R} \text { (Matching) } \\
\mathrm{R} \text { (Mismatching) } \\
\mathrm{C} \text { (Matching) } \\
\mathrm{C} \text { (Mismatching) } \\
\mathrm{S} \\
\mathrm{N}\end{array}$ & $\begin{array}{l}617 \\
685 \\
551 \\
577 \\
578 \\
588\end{array}$ & $\begin{array}{l}2.3 \\
3.1 \\
1.2 \\
1.4 \\
1.2 \\
1.7\end{array}$ & $\begin{array}{l}624 \\
697 \\
604 \\
622 \\
622 \\
639\end{array}$ & $\begin{array}{l}2.5 \\
2.9 \\
2.0 \\
1.8 \\
1.2 \\
1.9\end{array}$ & $\begin{array}{l}436 \\
473 \\
429 \\
448 \\
445 \\
462\end{array}$ & $\begin{array}{l}1.4 \\
2.0 \\
1.5 \\
2.3 \\
1.7 \\
2.4\end{array}$ & $\begin{array}{l}484 \\
522 \\
502 \\
518 \\
535 \\
514\end{array}$ & $\begin{array}{l}1.8 \\
2.2 \\
1.5 \\
2.5 \\
1.7 \\
2.5\end{array}$ \\
\hline
\end{tabular}


other conditions ( $p<.01$ in each case). The only other reliable difference between the conditions was a small benefit to RT on matching trials in Condition $\mathrm{C}$ in comparison with the matching trials in $\mathrm{R}$ and the no-distractor control, $N(p<.05$ in each case). In contrast, there were no differences between the conditions when the components differed in color.

The above effects could have been augmented by Stroop-type interference when the spatial position of the target (above vs. below fixation) differed with the required directional response (up vs. down; see Seymour, 1974). This possibility was tested in a further ANOVA with two between-subjects factors (component color and target color) and two withinsubjects factors (conditions and correspondence of directional response and field of target presentation). There was no Stroop-type effect $(F<1.0)$, and there were no reliable interactions between this variable and the other factors. This was also true of Experiments 2 and 3 , and consequently this factor will not be further discussed.

The percentage of errors in each condition were also collated. The general pattern of the data conformed to the RT results. In the same-color group, more errors were made in the random condition (R) than in the other conditions, while in the differentcolor group the number of errors were relatively consistent across the conditions (see Table 1). This indicates that the RT results were not confounded by a speed-accuracy tradeoff.

\section{Discussion}

The main finding is that the difference between the test conditions, $R$ and $C$, occurred only when the target and distractor were the same color. When the components differed in color, there were no differences between the conditions. This demonstrates that the reliable color difference between targets and distractors was sufficient to enable the selective processing of targets to take place.

The failure of subjects to selectively attend to targets in the same-color treatment replicates previous studies of the configural effect (Humphreys, 1981; Pomerantz \& Garner, 1973; Pomerantz \& Schwaitzberg, 1975). It is unlikely that this result occurred simply because subjects failed to attend to the location precued by the bar marker. Humphreys (1981) has shown that subjects can successfully attend to targets precued under the present conditions, providing that distractors are further than $1 \mathrm{deg}$ away from the cued location. This suggests that the result reflects a genuine lack of spatial selectivity within a 1-deg arc when subjects attend to nonfixated regions.

The effect of attending to both stimulus components (in the same-color treatment) was to impair performance when the distractor varied randomly (in $R$ ) relative to when it was constant (in C), straight (in S), or omitted (in N). The slower RTs in Condition $R$ were most likely due to response competition when both stimulus components were attended (cf. B. A. Eriksen \& C. W. Eriksen, 1974), as the impairment occurred only when targets and distractors mismatched in orientation. Response competition could have been avoided in the constant distractor condition (C) because subjects could respond to the stimulus configuration using a match/mismatch strategy (e.g., when the distractor was up across a block of trials, subjects could respond "up" when the components matched and "down" when they mismatched). Indeed, this is the strategy most subjects reported using in Condition $\mathrm{C}$. The facilitation effect on the matching trials in $C$ is consistent with this proposal, since subjects may make a "match" response between stimulus components from a rapid global comparison process (Bamber, 1969, 1972).

One rather puzzling finding, though, is the marked slower RTs in the no-distractor control condition, $\mathrm{N}$, for the same-color subject group relative to the different-color subject group (614 msec vs. $488 \mathrm{msec}$ over red and black targets, respectively; see Table 1). This condition was identical for the two color-congruity groups and consisted of a single target line presented in an otherwise blank visual field. Since color congruity was a between-subjects factor, the observed difference may have been due to sampling variation. However, the size of the effect suggests that this is unlikely. Alternatively, it could reflect differences in response criteria adopted by subjects in the samecolor and different-color groups as a function of their experience in the experimental conditions. B. A. Eriksen and C. W. Eriksen (1974) and C. W. Eriksen and B. A. Eriksen (1979) have both reported evidence that, when visual targets and distractors are presented in close spatial and temporal contiguity, response selection by target position requires subjects to use some form of inhibitory process to prevent responses being made to distractors. For example, subjects might perform a check of the component locations to ensure that they are not responding to distractors. In the same-color treatment here, subjects may have used such a strategy to prevent responses being made to distractors in the experimental conditions ( $R$ and $C$ ). If this strategy were carried over to the control conditions, slower RTs would result relative to when a less conservative criterion could be employed (in the different-color treatment). Indeed, same-color subjects who participated in the control conditions prior to the experimental conditions demonstrated quicker RTs in the no-distractor control, $\mathrm{N}$, than subjects who participated in the control conditions after the experimental conditions $(559 \mathrm{msec}$ 
vs. $632 \mathrm{msec}$ ). This suggests that the latter subjects carried over a conservative response criterion from the experimental to the control trials.

Since this conservative criterion would reflect the lack of spatial selectivity in the same-color treatment, the proposal accords with the argument that the selectivity of attention to targets in the different-color group was superior to that in the same-color group. However, one interesting implication is that the configural effect (the difference between Conditions $\mathbf{R}$ and C) may occur only when a conservative criterion is used. For instance, a check procedure may be quicker with a constant distractor, since subjects could respond from the relation between the stimuli (match vs. mismatch) rather than from their absolute locations in the visual field (as in Condition R). Experiment 3 assesses this proposal by using a response-deadline technique to prevent subjects from using conservative response criteria (cf. Pachella \& Pew, 1968; Pachella, Smith, \& Stanovich, 1978).

In addition to the above findings, there was a significant main effect of target color. This arose because subjects who responded to black targets were consistently faster than those who responded to red targets ( $522 \mathrm{msec}$ vs. $575 \mathrm{msec}$, over conditions and color congruity groups). A likely cause of this effect is the difference in the relative contrast of black and red components against the white-field background: black components were probably more salient than red components.

C. W. Eriksen and Schultz (1979) and Posner and Snyder (1975) have argued that response competition between stimuli will vary as a function of the relative speeds at which the competing stimuli are processed. Competition will increase when a target is processed slowly relative to a distractor, while it will be reduced when the target is processed relatively quickly. Accordingly, a color difference between the stimulus components could mitigate response competition effects if it speeds the processing of the target relative to the distractor. However, since black targets are responded to more quickly than red targets, facilitated target processing should occur only when the target is black and the distractor is red. Response competition may actually be increased (relative to when the stimuli are the same color) when the target is red and the distractor black. Contrary to this prediction, target color did not interact with either the color congruity or the condition factors. Indeed, inspection of the data in the different-color treatment (Table 1) suggests that the relative speed of processing targets and distractors had little effect on performance (e.g., the difference between RTs on matching and mismatching trials in $R$ was $38 \mathrm{msec}$ with red targets and black distractors and $37 \mathrm{msec}$ with black targets and red distractors). The color difference did not facilitate selective attention to targets simply by lessening their processing time relative to distractors.

We may now turn to the models of multidimensional stimulus processing presented in the introduction. At first sight, the explanation of the latter result also seems difficult for the independent dimensional processing account (e.g., Biederman \& Checkosky, 1970). This holds that RTs will be quicker when targets are specified on more than one dimension, because subjects may then respond on the basis of whichever dimension is resolved first. If there is an overlap in the resolution times of the location and color dimensions of the stimuli, any variable that affects the time to resolve the target on one dimension (e.g., target color) should also affect performance on the selective attention task. Clearly, this was not the case.

To explain the lack of an effect of target color on selective attention, the model must assume that even when the time to resolve the target's color was relatively slow (when the target was red), the color dimension was still resolved before the location dimension. This would avoid the interference effects that occurred in Condition $\mathbf{R}$ when subjects attended to the location dimension (in the same-color treatment), due to the lack of spatial selectivity. Furthermore, response competition between items specified solely by their color must be minimal. This would be the case if there was a higher specificity of attention along the color dimension (to the red or black target) than there was along the location dimension (to the cued target location).

The holistic processing model (Lockhead, 1972) can explain the findings with rather more ease. When the brackets differ in color, they will be represented further apart in multidimensional psychological space than when they are the same color. Consequently, targets will be selectively attended relatively more easily. Additionally, since selective attention is determined by the discriminability of components in this space, it will be independent of the absolute time taken to resolve the different colors. Performance will be as good when the target is red and the distractor black as when the reverse is true, since the discriminability of the components will be the same.

\section{EXPERIMENT 2}

Experiment 2 was designed to test between the independent dimensional processing and the holistic processing accounts of Experiment 1. It also examined whether the component color difference facilitated selective attention automatically or whether the effect was modulated by the subject's control processes.

Configurations from the set illustrated in Fig- 
ure $1 \mathrm{~b}$ were again presented to subjects, and targets were precued by a location bar marker. However, in this study, targets and distractors always differed in color (red vs. black), and the validity of color as a cue to the target was varied between subjects. For one group, the target was consistently one color (Group H; the high-validity condition); for a second group, the target was one color on $75 \%$ of the trials and the other color on $25 \%$ of the trials (Group $\mathrm{M}$; the medium-validity condition); and for a third group, the target was equally likely to be either color (Group L; the low-validity group).

If a component color difference facilitates selective attention automatically, no configural effects should be apparent in Experiment 2. That is, there should be no difference between the random and constant distractor conditions for any of the subject groups. On the other hand, if the color difference is effective only when it reliably specifies the target (as in Experiment 1), selective attention should be apparent in Group $\mathrm{H}$ but not in Group L. For instance, if we adapt the holistic processing model to accommodate perceiver-determined factors, subjects may be able to control the saliency of a particular dimension in psychological space. Stimulus color may not be salient when it does not consistently designate the target. If we, likewise, adapt the independent processing model, subjects may prevent attention being allocated to the color dimension in the low-validity condition.

In the medium-validity condition, both models predict that color information should be utilized, since it specifies the target on a majority of trials $(75 \%)$. On these trials, selective target processing should occur (as in the high-validity treatment). However, the models differ in their predictions of performance when the target's color differs from that expected (on $25 \%$ of the trials). The holistic processing model holds that selective attention depends on the discriminability of the component representations. Since discriminability will be constant irrespective of whether the target is colored as expected, targets should be attended with equal facility on the low $(25 \%)$ and on the high $(75 \%)$ probability trials in Group $M$. In contrast, the independent dimensional processing model predicts that subjects in Group $M$ will always attend to the color dimension prior to the location dimension. When the target is not colored as expected, subjects will (incorrectly) attend to the distractor. This should lead to a marked disruption of performance, particularly on trials in which the components mismatch in orientation in Condition $R$, when response competition is most marked.

\section{Method}

The same experimental paradigm and equipment as in the first study were employed.
Twenty-four paid volunteer subjects from the Birkbeck College subject pool took part (11 male, 13 female). All subjects had normal or corrected-to-normal vision and none had participated in Experiment 1.

The stimulus displays differed from the first study in that targets and distractors always differed in color, one being red and the other black. There were three independent groups of subjects (Groups H, M, and L). For half the subjects in Group H, the target was always red and the distractor black; the reverse was true for the other subjects. For half the subjects in Group $M$, the target was black and the distractor was red on $75 \%$ of the trials, and the target was red and the distractor black on the other $25 \%$ of the trials. This color probability assignment was reversed for the other subjects in Group M. For all the subjects in Group L, there was an equal probability of the target being red or black.

There were, again, four within-subjects conditions, in which the relation between the orientation of the target and the distractor was varied. The distractor could either be curved randomly across trials (Condition $\mathbf{R}$ ), curved in a constant direction across trials $(C)$, a straight line $(\mathrm{S})$, or omitted $(\mathrm{N})$. The conditions were presented in separate blocks to each subject, and the order of presentation of the blocks was counterbalanced over each subject group. There were 128 trials in each block, and the target was equally often above and below fixation and curved up and down (32 trials per combination). The order of presentation of the trials was pseudorandomized for each subject.

Participants were told at the outset of each block the likelihood that the target was in one particular color. They were asked to attend to the precued location and to respond to the orientation of the stimulus in that location as quickly as possible. The subjects were informed by the experimenter of any errors they had made.

\section{Results}

The data were collected using the same procedures as in the first experiment. Latencies \pm 3 standard deviations from each subject's mean correct RT in each condition were rejected, along with their error responses. The mean correct RTs and percentage errors for each color validity group in each condition are given in Table 2.

RTs were examinud in an ANOVA for a mixed design with one within-subjects factor (Conditions $R$ matching, $\mathrm{R}$ mismatching, $\mathrm{C}$ matching, $\mathrm{C}$ mismatching, $S$, and $N$ ), and one between-subjects factor (color validity). RTs were pooled across target color, because this factor was not distributed evenly across the subject groups (it was manipulated between subjects in Group $\mathrm{H}$ and within subjects in Group $\mathrm{L}$ ).

Table 2

Mean Reaction Times (RT) in Milliseconds and Percentage Errors (PE) in Each Color Validity Group as a Function of the Conditions in Experiment 2

\begin{tabular}{|c|c|c|c|c|c|c|}
\hline \multirow[b]{3}{*}{ Condition } & \multicolumn{6}{|c|}{ Color Validity Group } \\
\hline & \multicolumn{2}{|c|}{ High } & \multicolumn{2}{|c|}{ Medium } & \multicolumn{2}{|c|}{ Low } \\
\hline & RT & $\mathbf{P E}$ & RT & PE & RT & PE \\
\hline R (Matching) & 458 & .8 & 580 & 1.1 & 580 & 2.6 \\
\hline $\mathbf{R}$ (Mismatching) & 493 & 1.8 & 638 & 3.5 & 687 & 5.4 \\
\hline $\mathrm{C}$ (Matching) & 455 & 1.2 & 551 & 1.8 & 513 & 1.4 \\
\hline $\mathrm{C}$ (Mismatching) & 468 & 1.6 & 568 & 2.0 & 557 & 1.6 \\
\hline $\mathrm{S}$ & 481 & 1.7 & 585 & 1.4 & 544 & 1.0 \\
\hline $\mathbf{N}$ & 487 & 1.2 & 558 & 2.9 & 534 & 1.1 \\
\hline
\end{tabular}


The main effects of color validity and conditions were statistically significant $[F(2,21)=3.49, p<.05$ and $F(5,105)=48.14, p<.001$, respectively], as was the interaction between these factors $[F(10,105)=$ $12.78, \mathrm{p}<.001]$.

The Condition by Color validity interaction was analyzed in more detail using the Newman-Keuls technique. In Group $\mathrm{H}$, there was a small interference effect on mismatching trials in the random distractor condition (R) in comparison with the matching trials both in $\mathrm{R}$ and the constant distractor treatment, $C(p<.05$ in each case). In Group M, the impairment on the mismatching trials in $\mathrm{R}$ was more salient, as RTs were slower in this condition than in all the other treatments ( $p<.01$ in each case). A similar result obtained in Group $L(p<.01$ for the random mismatching condition relative to all the other conditions), for which performance was also facilitated on the matching trials in $C$ in comparison with the straight line control (S), the mismatching trials in $\mathrm{C}$, and the matching trials in $\mathrm{R}(\mathrm{p}<.01$ in each case). There were no other differences between the conditions in any of the groups.

In order to test the critical predictions of the independent dimensional processing and holistic processing models, performance in Group $M$ was further examined. Responses when targets were colored as expected (on $75 \%$ of the trials) and responses when targets were not colored as expected (on $25 \%$ of the trials) were separated. The mean correct RTs and the percentage errors in the target-color probability treatments as a function of the conditions and the expected target color are illustrated in Table 3.

An ANOVA for a mixed design was conducted on the RT data, with one between-subjects factor (expected target color) and two within-subjects factors (target color probability and conditions). The main effect of the conditions was statistically significant $[F(5,30)=18.26, p<.001]$, while the effects of expected target color and target color probability failed to reach significance $[F<1.0$ and $F(1,6)=3.60$, $\mathrm{p}>.05$, respectively]. There were also reliable inter- actions between target color probability and expected target color $[F(1,6)=70.78, p<.001]$, and between target color probability and the conditions $[F(5,30)=$ $6.42, \mathrm{p}<.001]$. All other interactions were nonsignificant $(\mathrm{F}<1.0)$.

A Newman-Keuls analysis of the interaction between target color probability and expected target color revealed that, in the high-probability treatment, RTs were slower when the expected target color was red than when it was black $(\mathrm{p}<.01)$, while this effect was reversed in the low-probability condition $(p<.01)$.

A similar analysis of the interaction between target color probability and conditions showed that there was a small impairment on the mismatching trials in $R$ relative to the constant matching treatment in the high $(75 \%)$ color probability condition $(p<.05)$. In the low $(25 \%)$ color probability condition, though, RTs were slower in the random mismatching treatment than in all the other conditions $(p<.01$ in each case). There were no other differences between the conditions at either color probability.

The error data went in the same direction as the RT results. For example, when performance is considered across the three color validity groups (Table 2), more errors were made as the validity of color as a cue to the target decreased. This trend was most marked on the mismatching trials in the random distractor condition (R). Similarly, in Group $\mathbf{M}$ most errors were generated in the $\mathbf{R}$ mismatching condition, particularly when there was a low probability for the target's color. The equivalence of the RT and error results indicates that latencies in any one condition were not traded off with classification accuracy.

\section{Discussion}

The color difference between the components did not facilitate selective attention automatically. Selective attention, measured by the difference between Conditions $\mathbf{R}$ and $\mathrm{C}$, was superior in Group $\mathbf{H}$ relative to Group $\mathbf{M}$, and in Group $\mathbf{M}$ relative to Group L.

Table 3

Mean Correct Reaction Times (RT) in Milliseconds and Percentage Errors (PE) in Each Condition as a Function of the Expected Target Color (ETC) and the Probability of the Target Color in Group M in Experiment 2

Probability of Target Color

\begin{tabular}{|c|c|c|c|c|c|c|c|c|}
\hline \multirow[b]{3}{*}{ Condition } & \multicolumn{4}{|c|}{.75} & \multicolumn{4}{|c|}{.25} \\
\hline & \multicolumn{2}{|c|}{ ETC: Black } & \multicolumn{2}{|c|}{ ETC: Red } & \multicolumn{2}{|c|}{ ETC: Black } & \multicolumn{2}{|c|}{ ETC: Red } \\
\hline & RT & $\mathrm{PE}$ & RT & $\mathrm{PE}$ & RT & PE & RT & PE \\
\hline R (Matching) & 504 & .8 & 643 & 1.0 & 662 & 1.1 & 509 & 1.2 \\
\hline R (Mismatching) & 515 & 1.6 & 679 & 2.2 & 742 & 5.7 & 613 & 4.5 \\
\hline C (Matching) & 464 & 1.2 & 619 & 1.2 & 625 & 2.6 & 495 & 2.2 \\
\hline C (Mismatching) & 484 & 1.0 & 628 & 1.6 & 653 & 3.1 & 508 & 2.9 \\
\hline S & 475 & 1.5 & 647 & 1.6 & 672 & 2.4 & 525 & 2.3 \\
\hline $\mathrm{N}$ & 481 & .7 & 625 & .9 & 624 & 2.0 & 498 & 2.0 \\
\hline
\end{tabular}


Thus, selective attention improved as a function of the likelihood that the target was specified by its color. This demonstrates that the effects of stimulus quality (the color difference between targets and distractors) are modulated by the perceiver, since their utility is dependent on the task demands. In order to explain this result, the independent dimensional processing and the holistic processing models must be adapted to allow control procedures to influence stimulus analysis. Two possible adaptations were discussed earlier.

The critical test between these models concerns their predictions of performance on the low target color probability trials in Group $M$. The holistic processing model expects no differences between performance on these trials relative to the high target color probability trials. The independent dimensional processing model predicts worse selective attention on the low than on the high target color probability trials. The results unequivocally support the latter model. Selective attention was considerably more impaired on trials in which the target's color had a $25 \%$ probability of occurrence than on those in which it had a $75 \%$ probability.

It seems, from this, that subjects can attend independently to the color and location of stimuli and, furthermore, that they can control their attention to either dimension. When color is not informative (as in Group $L$ here), subjects attend only to the location dimension. The selective processing of targets is then relatively poor due to the lack of spatial selectivity in the present circumstances. When color is informative (as in Groups $H$ and $M$ ), attention is exclusive to stimulus color. The processing of targets that are correctly specified by their color will suffer little interference from distractors because of the specificity of attention along the color dimension. However, on the small proportion of trials when targets are incorrectly specified by their color (in the low color probability treatment), subjects will attend to the distractor (since that is in the expected target color). This produced marked impairments of performance on mismatching trials in Condition $\mathbf{R}$. Since impairments were manifested mainly as slow, but correct, responses, it seems that the component locations were resolved prior to a response being made (otherwise, subjects would only make errors on mismatching trials). This suggests that a location-check operation was carried out after the stimuli had been parsed as targets and distractors on the basis of their color in Group M. This procedure will be less efficient when subjects attend to the distractor (on the low color probability trials) than when they attend to the target (on the high color probability trials).

We have argued, from the results of Experiment 1, that a location-check operation may also be neces- sary when subjects attend solely to the locations of the stimuli (in Group L here). This would not be the case in the high color validity treatment (Group H), as color could then be attended without any costs. If a location-check procedure was carried across from the experimental to the control trials in the relavant conditions, there should be differences between the color validity groups even when targets were presented in isolation (in the no-distractor control, $\mathbf{N}$ ). Consistent with this proposal, there were marked differences between the groups in this condition (487 msec for Group H vs. $558 \mathrm{msec}$ for Group M and $534 \mathrm{msec}$ for Group L; see Table 2). Unfortunately, color validity was manipulated between subjects, so this difference could simply reflect a sampling variation (cf. Experiment 1). Nevertheless, the size of the effect is suggestive of a genuine criterion difference between the groups. It remains possible that the difference between the experimental conditions $\mathbf{R}$ and $\mathrm{C}$ occurred primarily because the location check was facilitated when the distractor was constant relative to when it varied randomly in orientation.

In addition to the interaction between target color probability and conditions in Group $\mathbf{M}$, there was also an interaction between target color probability and expected target color. This occurred because black targets were responded to more quickly than red targets. In the high color probability treatment, expected black targets were discriminated more efficiently than expected red targets. In the low color probability treatment (when targets were in the unexpected color), RTs were slower when targets were expected to be black (and were red) than vice versa. The finding is consistent with the main effect of target color in Experiment 1, and was most probably due to the higher contrast of black targets. As in the first study, the color effect did not interact with the other treatments. Thus interference was not a function of the relative processing times of targets and distractors along the color dimension.

\section{EXPERIMENT 3}

One way to prevent subjects from using a conservative response strategy in a $R T$ study is to use a response-deadline technique (cf. Pachella \& Pew, 1968; Pachella et al., 1978). This requires subjects to attempt to respond prior to a specified criterion time and for error rate to be used as an index of processing difficulty. If a short deadline is used, any tendency to use a conservative response strategy (e.g., checking component locations) should be reduced.

Experiment 3 used a response-deadline technique to examine the present configural effect (the difference between the experimental conditions, $R$ and $C$ ). 
As in the medium color validity treatment of Experiment 2 (Group M), targets and distractors always differed in color and the target was specified by its color on $75 \%$ of the trials. However, the subjects were required to respond using a short deadline criterion. In this case, responses should be determined by the fastest dimension to be resolved. If this is stimulus color, performance should be relatively accurate when targets are specified by their color (on the high color probability trials) and inaccurate when targets are incorrectly specified by their color (on the low color probability trials). Additionally, if the configural effect occurs only because a location-check procedure is more accurate when the distractor is constant in orientation, it should be minimized when subjects respond to stimulus color alone.

\section{Method}

Unless otherwise mentioned, the apparatus, design, and procedure were the same as in Experiments 1 and 2.

Six volunteer subjects from the Birkbeck College subject pool participated (four male, two female). All had normal or correctedto-normal vision and all had taken part in Experiment 2. The subjects undertook four experimental sessions, each lasting about $50 \mathrm{~min}$.

The subjects were first given practice with the response-deadline procedure. They were asked to attend to the precued location and to respond to the curvature of the stimulus in that location before a criterion time was reached. They were informed of the deadline prior to the onset of the experimental trials, and they were given verbal feedback concerning their speed of response after every trial. The feedback was the word "fast" or the word "slow," depending on whether the response was made before or after the required deadline. The deadline for each subject was established during the practice trials. These were run in blocks of 10 , during which the distractor was a straight line. The experimenter varied the deadline in the range $250-350 \mathrm{msec}$, and practice was continued until a deadline was established which subjects could consistently match while making about $20 \%$ errors. This required a mean of 10 practice blocks over subjects, and there was a range of between 8 and 12 practice blocks. This procedure was carried out in each session undertaken by the subjects.

The two control conditions ( $\mathrm{S}$ and $\mathrm{N}$ ) were omitted in this study, and any differences between the experimental conditions ( $R$ and $C$ ) were assumed to reflect mainly interference in Condition $\mathbf{R}$. For half the subjects, the target was black and the distractor red on $75 \%$ of the trials and the target red and distractor black on $25 \%$ of the trials. It was the reverse for the other subjects. The sub- jects were told the likelihood of the target color prior to each session.

The conditions were presented in separate sessions, and the last two sessions undertaken by each subject counterbalanced the random order of the conditions in the first two sessions. This was to provide a check on whether practice differentially affected performance in the conditions. There were 128 experimental trials in each session (256 per condition), and the target was equally often above and below fixation and curved up and down ( 32 trials per combination).

\section{Results}

RTs \pm 3 standard deviations from each subject's mean correct latency in each treatment were discarded, along with the error responses. Table 4 presents the mean correct RTs and percentage of errors in each condition as a function of the target color probability and the expected target color.

RTs were analyzed in an ANOVA for a mixed design. There was one between-subjects factor, expected target color. There were four within-subjects factors: target color probability (.75 and .25), conditions ( $R$ and $C$ ), target-distractor congruence (matching and mismatching), and practice (first two sessions and last two sessions). The only statistically significant main effect was that of practice $[F(1,4)=11.22$, $\mathrm{p}<.05]$. There was also a reliable interaction between expected target color and target color probability $[F(1,4)=30.25, p<.01]$. None of the other interactions approached statistical significance.

Further analysis of the interaction between expected target color and target color probability was conducted using the Newman-Keuls method. In the high-probability treatment, RTs to expected black targets were quicker than RTs to expected red targets $(p<.01)$. In contrast, in the low-probability treatment. RTs were quicker when red targets were expected than when black targets were expected $(p<.01)$.

The accuracy data were analyzed using the same methods. The main effects of target color probability, target-distractor congruence, and practice were all statistically significant $[\mathrm{F}(1,4)=32.42, \mathrm{~F}(1,4)$ $=66.93$, both ps $<.01 ;$ and $F(1,4)=11.17, \mathrm{p}<.05$, respectively). The effects of expected target color and

Table 4

Mean Correct Reaction Times (RT) in Milliseconds and Percentage Errors (PE) as a Function of the Target Color Probability, the Expected Target Color (ETC), and the Conditions in Experiment 3

\begin{tabular}{|c|c|c|c|c|c|c|c|c|}
\hline \multirow{4}{*}{ Condition } & \multicolumn{8}{|c|}{ Probability of Target Color } \\
\hline & \multicolumn{4}{|c|}{.75} & \multicolumn{4}{|c|}{.25} \\
\hline & \multicolumn{2}{|c|}{ ETC: Black } & \multicolumn{2}{|c|}{ ETC: Red } & \multicolumn{2}{|c|}{ ETC: Black } & \multicolumn{2}{|c|}{ ETC: Red } \\
\hline & RT & $\mathrm{PE}$ & RT & $\mathrm{PE}$ & RT & PE & RT & $\mathbf{P E}$ \\
\hline R (Matching) & 255 & 8.4 & 294 & 21.0 & 292 & 21.4 & 271 & 14.3 \\
\hline $\mathrm{R}$ (Mismatching) & 253 & 23.7 & 296 & 22.4 & 323 & 62.7 & 253 & 58.7 \\
\hline C (Matching) & 260 & 11.1 & 294 & 23.7 & 284 & 25.4 & 264 & 14.5 \\
\hline C (Mismatching) & 266 & 15.4 & 300 & 11.7 & 313 & 50.4 & 280 & 50.0 \\
\hline
\end{tabular}


conditions did not approach significance (both Fs $<1.0$ ). The only reliable interactions were between target color probability and target-distractor congruence $[F(1,4)=24.68, p<.01]$ and between target color probability and practice $[\mathrm{F}(1,4)=28.26, \mathrm{p}<.01]$.

Analysis of the first interaction indicated that, in the low color probability treatment, responses were more accurate when the target and distractor matched than when they mismatched in orientation $(p<.01$, Newman-Keuls). In contrast, there was no effect of target-distractor congruence in the high color probability treatment.

A similar analysis of the Target Color Probability by Practice interaction showed that there was no effect of practice in the high color probability treatment, while responses were more accurate in the low color probability treatment on the first two experimental sessions than on the second two sessions ( $\mathrm{p}<.01$, Newman-Keuls).

\section{Discussion}

The response-deadline technique was successful in producing RTs that were considerably faster than those obtained in the earlier studies. For instance, average RTs were quicker by $304 \mathrm{msec}$ in Experiment 3 than in the equivalent conditions in Group $M$ in Experiment 2. In this circumstance, subjects should have been less able to use a conservative response strategy. Nevertheless, there was a similar effect of target color to that found in Experiments 1 and 2 . Black targets were responded to more quickly than red targets, and this generated an interaction between the expected target color and the target color probability factors. It seems that this color effect is not dependent solely on the response strategy, and that it is due to differences in the resolution times for red and black stimuli.

In contrast, there were no configural effects. Performance was equivalent in the experimental conditions, $R$ and $C$, on both $R T$ and response accuracy measures. This suggests that the configural effects obtained in Experiments 1 and 2 stemmed from the conservative response strategy adopted by subjects (e.g., making a location check before responding). When the utility of this strategy is reduced, configural effects are minimized.

In both experimental conditions ( $\mathrm{R}$ and $\mathrm{C}$ ), more errors were made when the target and distractor mismatched in orientation than when they matched, but only when the target's color had a low probability of occurrence. This finding is consistent with the proposal that subjects tended to respond to the component in the expected target color without carrying out a location check. Fast, correct responses should be made when targets are colored as expected (in the high-probability treatment) and when targets are not colored as expected but the distractor is curved in the same direction as the target (in the low-probability treatment). However, a high proportion of errors would result in the low-probability treatment when the stimuli differ in orientation.

The amount of experience subjects had at the task affected both RT and accuracy performance. Responses were made more quickly, the more practiced subjects became. Additionally, more errors tended to be made in the low color probability treatment. This indicates that subjects became less conservative with practice and were more prepared to respond solely on the basis of stimulus color. This would precipitate more errors when the target was not colored as expected.

\section{GENERAL DISCUSSION}

Three experiments have examined the effects of stimulus variables on selective visual attention. Subjects were precued to the location of a target line whose orientation they had to judge. Selective attention was assessed by the effects on target responses produced by distractors presented in close spatial proximity. In particular, selective attention was assumed to be impaired when there was a difference in performance when the distractor varied randomly in orientation relative to when it was constant (cf. Pomerantz \& Garner, 1973).

In Experiment 1, subjects were unable to selectively attend to targets that were the same color as distractors. In this circumstance, attention was to the target-distractor configuration, presumably because of poor spatial selectivity between the components. However, a reliable color difference between the stimulus components enabled targets to be selectively processed. Experiment 2 demonstrated that the color differences did not facilitate selective processing automatically, since selective attention occurred only when targets were consistently specified by their color. Furthermore, an informative color difference appeared to be effective because the target's color could be resolved more efficiently than its location, and subjects were able to attend exclusively to the color dimension. Thus, RTs were markedly slower when the target was in the expected distractor color and the distractor was in the expected target color. Errors could be avoided by subjects' checking the component locations prior to responding. Experiment 3 employed a response-deadline technique to examine this possibility. Under the deadline restrictions, subjects were assumed to respond to the first informative dimension to be resolved without a checking procedure being effected. The study confirmed that stimulus color was resolved first, since a high proportion of errors were produced when distractors were in the expected target color and differed 
in orientation relative to the target. Additionally, the difference between the random and constant distractor conditions was minimized under the fast deadline requirements. This suggests that this difference occurs because a location check is facilitated when the distractor is constant in orientation relative to when it varies randomly. When subjects attend only to stimulus location (cf. the same-color condition of Experiment 1), such a check may be necessary to avoid responses to distractors in close spatial proximity (B. A. Eriksen \& C. W. Eriksen, 1974; C. W. Eriksen \& B. A. Eriksen, 1979).

These results strongly support an independent dimensional processing model of perception (Biederman \& Checkosky, 1970; Hawkins, 1969). This assumes that increasing stimulus quality by varying targets and distractors along two rather than one dimension facilitates selective attention when targets can be resolved more efficiently by means of the second dimension than by means of the first. In the present study, the color difference between the components produced selective target processing because stimulus color was resolved more quickly and more accurately than stimulus location. However, since this occurred only when stimulus color was informative (Experiment 2), it seems that subjects have some control over the utility of independently processed stimulus information. In particular, they seem able to prevent attention from being allocated to any dimension that is unlikely to specify the target.

The question arises as to whether a similar flexibility of attention between stimulus dimensions can be demonstrated with other arbitrary stimulus dimensions (Biederman \& Checkosky, 1970; Dykes, 1979) or whether this depends on the nature of the stimulus (Garner, 1974). For instance, subjects may be able to attend optionally and exclusively between stimulus color and location only because these dimensions are separable (Garner, 1970, 1974) or asymmetrically integral (i.e., they allow selective attention to color but not to location; cf. Garner, 1976; Geller, 1977). This may not be the case with integral stimulus dimensions, such as saturation and brightness (Garner \& Felfoldy, 1970; Smith \& Kemler, 1978). Studies of the flexibility of attention between different combinations of stimulus dimensions should provide further insights into the interaction between stimulusdetermined and perceiver-determined factors in perception.

\section{REFERENCES}

Allport, D. A. Parallel encoding within and between elementary stimulus dimensions. Perception \& Psychophysics, 1971, 10, 104-108.

Allport, D. A. Attention and performance. In G. L. Claxton (Ed.), New directions in cognitive psychology. London: Routledge \& Kegan Paul, 1980.
Bamber, D. Reaction times and error rates for "same"-."different" judgments of multidimensional stimuli. Perception \& Psychophysics, 1969, 6, 169-174.

BAMBER, D. Reaction times and error rates for judging nominal identity of letter strings. Perception \& Psychophysics, 1972, 12, 321-326.

Biederman, I., \& Checkosky, S. F. Processing redundant information. Journal of Experimental Psychology, 1970, 83, 486-490.

Chen, M. J., \& Allnutr, P. Abstraction of information from a complex display. Perception, 1977, 6, 377-391.

Clark, S. E. Retrieval of color information from preperceptual memory. Journal of Experimental Psychology, 1969, 82, 263-266.

Colegate, R. L., Hoffman, J. E., \& Eriksen, C. W. Selective encoding from multielement visual displays. Perception \& Psychophysics, 1973, 14, 217-224.

Duncan, J. The demonstration of capacity limitation. Cognitive Psychology, 1980, 12, 75-96.

Dykes, J. R., JR. A demonstration of selection of analyzers for integral dimensions. Journal of Experimental Psychology: Human Perception and Performance, 1979, 5, 734-745.

Dykes, J. R., JR., \& CoOper, R. G. An investigation of the perceptual basis of redundancy gain and orthogonal interference for integral dimensions. Perception \& Psychophysics, 1978, 23, 36-42.

Eriksen, B. A., \& Eriksen, C. W. Effects of noise letters upon the identification of a target letter in a nonsearch task. Perception \& Psychophysics, 1974, 16, 143-149.

Eriksen, C. W., \& Eniksen, B. A. Target redundancy in visual search: Do repetitions of the target within the display impair processing? Perception \& Psychophysics, 1979, 26, 195-205.

Eriksen, C. W., Hamlin, R. M., \& Daye, C. The effect of flanking letters and digits on speed of identifying a letter. Bulletin of the Psychonomic Society, 1973, 2, 400-402.

Eriksen, C. W., \& Hoffman, J. E. Some characteristics of selective attention in visual perception determined by vocal reaction time. Perception \& Psychophysics, 1972, 11, 169-171.

Eriksen, C. W., \& Hofrman, J. E. The extent of processing of noise elements during selective encoding from visual displays. Perception \& Psychophysics, 1973, 14, 155-160.

Eriksen, C. W., \& Schultz, D. W. Information processing in visual search: A continuous flow conception and experimental results. Perception \& Psychophysics, 1979, 25, 249-263.

GarNer, W. $R$. The stimulus in information processing. American Psychologist, 1970, 25, 350-358.

GARNER, W. R. The processing of information and structure. Potomac, Md: Erlbaum, 1974.

GARNER, W. R. Interaction of stimulus dimensions in concept and choice processes. Cognitive Psychology, 1976, 8, 98-123.

Garner, W. R., \& Felfoldy, G. L. Integrality of stimulus dimensions in various types of information processing. Cognitive Psychology, 1970, 1, 225-241.

Geller, E. S. Latencies to name one of three stimulus dimensions: A study of probability effects and dimension integrality. Perception \& Psychophysics, 1977, 22, 70-76.

Hawkins, H. L. Parallel processing in complex visual discrimination. Perception \& Psychophysics, 1969, 5, 45-64.

HUMPHREYs, $G$. W. On varying the span of visual attention: Evidence for two modes of spatial attention. Quarterly Journal of Experimental Psychology, 1981, 33A, 17-31.

LOCKHEAD, G. R. Effects of dimensional redundancy on visual discrimination. Journal of Experimental Psychology, 1966, 72, 95-104.

LOCKHEAD, G. R. Identification and the form of multidimensional discrimination space. Journal of Experimental Psychology, 1970, 85, 1-10.

Lockhead, G. R. Processing dimensional stimuli: A note. Psychological Review, 1972, 79, 410-419.

LockheaD, G. R., \& King, M. C. Classifying integral stimuli. 
Journal of Experimental Psychology: Human Perception and Performance, 1977, 3, 436-443.

Mathews, M. L. Facilitation of letter recognition by an annulus in a metacontrast paradigm. Quarterly Journal of Experimental Psychology, 1974, 26, 312-323.

Nickerson, R. S. Comment on W. R. Garner's 'Selective attention to attributes and to stimuli.' Journal of Experimental Psy. chology: General, 1978, 107, 452-456.

Norman, D. A., \& Boвrow, D. G. On data-limited and resourcelimited processes. Cognitive Psychology, 1975, 7, 44-64.

Pachella, R. G., \& Pew, R. W. Speed-accuracy tradeoff in reaction time: Effect of discrete criterion times. Journal of Experimental Psychology, 1968, 76, 19-24.

Pachella, R. G., Smith, J. E. K., \& Stanovich, K. E. Qualitative error analysis and speeded classification. In J. Castellan \& F. Restle (Eds.), Cognitive theory (Vol. 3). Hillsdale, N.J: Erlbaum, 1978.

Pomerantz, J. R., \& Garner, W. R. Stimulus configurations in selective attention tasks. Perception \& Psychophysics, 1973, 14. $565-569$.

Pomerantz, J. R., \& Schwaitzberg, S. D. Grouping by prox- imity: Selective attention measures. Perception \& Psychophysics, $1975,18,355-361$.

Posner, M. I., \& Snyder, C. R. R. Attention and cognitive control. In R. L. Solso (Ed.), Information processing and cognition: The Loyola symposium. Hillsdale, N.J: Erlbaum, 1975.

SEymour, P. H. K. Stroop interference with response competition, comparison and encoding stages in a sentence-picture comparison task. Memory \& Cognition, 1974, 2, 19-26.

Shaw, P. Processing of tachistoscopic displays with controlled order of characters and spaces. Perception \& Psychophysics, 1969, 6, 257-266.

Smith, L. B., \& Kemlen, D. G. Levels of experienced dimensionality in children and adults. Cognitive Psychology, 1978, 10, 502-532.

Williams, L. G. The effect of target specification on objects fixated during visual search. Perception \& Psychophysics, 1966, 1, 315-318.

(Received for publication December 18, 1980; accepted April 29, 1981.) 